\title{
SOME INEQUALITIES FOR QUANTUM TSALLIS ENTROPY RELATED TO THE STRONG SUBADDITIVITY
}

\author{
DÉnes Petz And DÁNiel ViRoszTeK
}

\begin{abstract}
In this paper we investigate the inequality $S_{q}\left(\rho_{123}\right)+S_{q}\left(\rho_{2}\right) \leqslant S_{q}\left(\rho_{12}\right)+S_{q}\left(\rho_{23}\right)(*)$ where $\rho_{123}$ is a state on a finite dimensional Hilbert space $\mathscr{H}_{1} \otimes \mathscr{H}_{2} \otimes \mathscr{H}_{3}$, and $S_{q}$ is the Tsallis entropy. It is well-known that the strong subadditivity of the von Neumnann entropy can be derived from the monotonicity of the Umegaki relative entropy. Now, we present an equivalent form of $(*)$, which is an inequality of relative quasi-entropies. We derive an inequality of the form $S_{q}\left(\rho_{123}\right)+S_{q}\left(\rho_{2}\right) \leqslant S_{q}\left(\rho_{12}\right)+S_{q}\left(\rho_{23}\right)+f_{q}\left(\rho_{123}\right)$, where $f_{1}\left(\rho_{123}\right)=0$. Such a result can be considered as a generalization of the strong subadditivity of the von Neumnann entropy. One can see that $(*)$ does not hold in general (a picturesque example is included in this paper), but we give a sufficient condition for this inequality, as well.
\end{abstract}

Mathematics subject classification (2010): 46N50, 94A15, 46L30, 47L90.

Keywords and phrases: Tsallis entropy, strong subadditivity.

\section{REFERENCES}

[1] J. ACZÉL AND Z. DARóCZy, On Measures of Information and Their Characterizations, Academic Press, San Diego, 1975.

[2] H. ARAKI, Relative entropy of state of von Neumann algebras, Publ. RIMS Kyoto Univ. 9 (1976), 809-833.

[3] K. M. R. Audenaert, Subadditivity of q-entropies for $q>1$, J. Math. Phys. 48 (2007), 083507.

[4] Á. Besenyei And D. Petz, Partial subadditivity of entropies, Linear Algebra and its Applications, 439 (2013), 3297-3305.

[5] R. Bhatia, Matrix analysis, Springer, 1996.

[6] E. CARLEN, Trace inequalities and quantum entropy: an introductory course, Contemp. Math. 529 (2010), 73-140.

[7] Z. DARÓCZI, General information functions, Information and Control, 16 (1970), 36-51.

[8] E. Effros, A Matrix Convexity Approach to Some Celebrated Quantum Inequalities, Proc. Natl. Acad. Sci. USA, 106 (2009), 1006-1008.

[9] S. FURUICHI, Information theoretical properties of Tsallis entropies, J. Math. Phys. 47, 023302 (2006).

[10] S. Furuichi, K. YANAGi And K. Kuriyama, Fundamental properties of Tsallis relative entropy, J. Math.Phys. 45 (2004), 4868-4877.

[11] G. H. Hardy, J. E. Littlewood and G. Pólya, Inequalities, Cambridge University Press, Cambridge, 1934.

[12] F. Hiai And D. Petz, From quasi-entropy to various quantum information quantities, Publ. RIMS Kyoto University 48 (2012), 525-542.

[13] F. Hiai And D. Petz, Introduction to Matrix Analysis and Applications, Hindustan Book Agency and Springer Verlag, 2014.

[14] A. JENČOVÁ AND M. B. RUSKAI, A unified treatment of convexity of relative entropy and related trace functions, with conditions for equality, Rev. Math. Phys. 22 (2010), 1099-1121.

[15] I. H. Kim, Operator extension of strong subadditivity of entropy, J. Math. Phys. 53 (2012), 122204.

[16] E. Lieb And M. B. Ruskai, Proof of the strong subadditivity of quantum-mechanical entropy, J. Math. Phys. 14 (1973), 1938-1941. 
[17] M. Nielsen AND D. PetZ, A simple proof of the strong subadditivity inequality, Quantum Information \& Computation, 6 (2005), 507-513.

[18] M. Ohya AND D. PetZ, Quantum Entropy and its Use, Springer-Verlag, Berlin, 1993.

[19] D. PetZ, Quasi-entropies for finite quantum systems, Rep. Math. Phys. 23 (1986), 57-65.

[20] D. Petz AND D. ViroszteK, A characterization theorem for matrix variances, to appear in Acta Sci. Math. (Szeged), in 2014.

[21] N. Sharma, Equality Conditions for Quantum Quasi-Entropies Under Monotonicity and JointConvexity, Nat. Conf. Commun. (NCC), 2014. 